\title{
Data assimilation for massive autonomous systems based on a second-order adjoint method
}

\author{
Shin-ichi Ito, ${ }^{1}$ Hiromichi Nagao, ${ }^{1,2}$ Akinori Yamanaka, ${ }^{3}$ Yuhki Tsukada, ${ }^{4}$ Toshiyuki Koyama, ${ }^{4}$ \\ Masayuki Kano, ${ }^{1}$ and Junya Inoue ${ }^{5}$ \\ ${ }^{1}$ Earthquake Research Institute, The University of Tokyo, 1-1-1, Yayoi, Bunkyo-ku, Tokyo 113-0032, Japan \\ ${ }^{2}$ Graduate School of Information Science and Technology, The University of Tokyo, 7-3-1, Hongo, Bunkyo-ku, 113-8656 Tokyo, Japan \\ ${ }^{3}$ Division of Advanced Mechanical Systems Engineering, Institute of Engineering, \\ Tokyo University of Agriculture and Technology, 2-24-16, Naka-cho, Koganei, Tokyo 184-8588, Japan \\ ${ }^{4}$ Graduate School of Engineering, Nagoya University, Furo-cho, Chikusa-ku, Nagoya, Aichi 464-8603, Japan \\ ${ }^{5}$ Research Center for Advanced Science and Technology, The University of Tokyo, 4-6-1, Komaba, Meguro-ku, Tokyo 153-8904, Japan
}

(Received 14 March 2016; revised manuscript received 25 July 2016; published 14 October 2016)

\begin{abstract}
Data assimilation (DA) is a fundamental computational technique that integrates numerical simulation models and observation data on the basis of Bayesian statistics. Originally developed for meteorology, especially weather forecasting, DA is now an accepted technique in various scientific fields. One key issue that remains controversial is the implementation of DA in massive simulation models under the constraints of limited computation time and resources. In this paper, we propose an adjoint-based DA method for massive autonomous models that produces optimum estimates and their uncertainties within reasonable computation time and resource constraints. The uncertainties are given as several diagonal elements of an inverse Hessian matrix, which is the covariance matrix of a normal distribution that approximates the target posterior probability density function in the neighborhood of the optimum. Conventional algorithms for deriving the inverse Hessian matrix require $O\left(C N^{2}+N^{3}\right)$ computations and $O\left(N^{2}\right)$ memory, where $N$ is the number of degrees of freedom of a given autonomous system and $C$ is the number of computations needed to simulate time series of suitable length. The proposed method using a second-order adjoint method allows us to directly evaluate the diagonal elements of the inverse Hessian matrix without computing all of its elements. This drastically reduces the number of computations to $O(C)$ and the amount of memory to $O(N)$ for each diagonal element. The proposed method is validated through numerical tests using a massive two-dimensional Kobayashi phase-field model. We confirm that the proposed method correctly reproduces the parameter and initial state assumed in advance, and successfully evaluates the uncertainty of the parameter. Such information regarding uncertainty is valuable, as it can be used to optimize the design of experiments.
\end{abstract}

DOI: 10.1103/PhysRevE.94.043307

\section{INTRODUCTION}

Determining the model parameters and initial states of simulation models is an important task in various scientific fields, as it enables us to predict the temporal evolution of the target system. However, in many practical cases, this procedure is somewhat complex, because some of the parameters and initial states cannot always be directly observed by experiments. In materials science, for example, phase-field (PF) models are often used to simulate the evolution of microstructures during the processes of solidification and phase transformation [1-7]. PF models phenomenologically describe the dynamics of phases using field variables that evolve in time depending on the gradient of the total free energy. Since a PF model usually requires a huge number of grid points to discretize the field variables, the computational cost tends to be prohibitive. Nonetheless, PF models are accepted beyond the field of materials science, such as in hydrodynamics [8-10], because they can be employed to model phases and their dynamics using mathematical expressions that are easy to manipulate. Since the microstructure of materials such as steel, stainless and aluminium alloys affects the mechanical properties, controlling the temporal evolution of the microstructure is a key issue in obtaining the desired mechanical properties. The PF simulations help us to understand and predict the dynamics of the microstructure for the efficient development of real materials. The problem to be solved is to determine the initial state and parameters involved in a PF model, which are usually unobservable directly by experiments. This parameter estimation is practically impossible because of the computational cost of forward simulations and/or insufficient experimental data. However, recent developments in supercomputers and enhancements in experimental equipment have enabled the parameter estimation process to begin by integrating simulations and observations, which is a longstanding problem in materials science. This paper focuses on parameter estimation in the framework of data assimilation (DA), implementing a new method to quantify the uncertainties of the estimates, and thus providing valuable feedback for experimental design.

DA is a computational technique that integrates numerical simulation models and observational data on the basis of Bayesian statistics. Thus, DA enables the parameters and initial states of PF models to be estimated by systematically extracting as much information as possible from the given observational and experimental data. The process of DA evaluates a probability density function (PDF) (or the "posterior PDF," to be precise) of the unknown parameters and unobservable states that is conditional on the given observation data [11]. DA was originally developed in the fields of meteorology and oceanography [12-14], but is now applied in areas such as seismology, marketing science, and industrial science [15-18]. Several sequential Bayesian filters 
and other nonsequential estimation methods have been used in DA. Common sequential Bayesian filters such as the ensemble Kalman filter [19-21] and the particle filter [22-24] estimate the target posterior PDF using Bayes' theorem. This approximation is formed using an ensemble of realizations, meaning that the computational cost is proportional to the number of realizations. The implementation of one sequential Bayesian filter on a given simulation model is not especially complex, and a sufficiently accurate estimate of the posterior PDF can be achieved when the number of degrees of freedom $N$ of the simulation model is sufficiently small. However, Bayesian filters become inefficient when applied to massive simulation models, as the number of realizations required to obtain a converged posterior PDF is proportional to $e^{O(N)}$.

Unlike sequential Bayesian filters, adjoint methods [25-27] directly determine the optimum solution using a gradient method to maximize the target posterior PDF. Although this achieves a drastic reduction in the computational cost, the ordinary adjoint method cannot evaluate the uncertainty in its estimations, which sequential Bayesian filters obtain in a straightforward manner. Such uncertainties provide valuable information related to both the estimations and the optimum solution. For example, the uncertainties provide feedback for the experimental design that helps to identify the parameters of interest with the required accuracy. The quantification of uncertainty is currently a very important issue in the application of DA to massive simulation models. The uncertainty is generally represented by the second-order cumulant of the posterior PDF, i.e., covariance matrix $\boldsymbol{\Sigma}$, which can be approximated by the inverse of the second-order derivative of the logarithmic posterior PDF, i.e., inverse of the Hessian matrix $\boldsymbol{H}^{-1}$. Since the calculation of $\boldsymbol{\Sigma}$ or $\boldsymbol{H}^{-1}$ is computationally expensive, the first-order derivative of the logarithmic posterior PDF, i.e., sensitivity, has been widely used instead of $\boldsymbol{\Sigma}$ or $\boldsymbol{H}^{-1}$ to investigate how the PDF changes with respect to the variables of interest [28,29]. Recent technological developments in computers have motivated us to directly evaluate $\boldsymbol{\Sigma}$ or $\boldsymbol{H}^{-1}$. However, previous studies attempting this presupposed the computation of all elements in the matrix, and thus imposed some limitations such as the small dimension of observations [30], the tangent-linear approximation for given simulation models [31], or an approximate computation of the matrix using a quasi-Newton method [32-34].

In many cases, not all elements in the matrix are of interest, and knowing only a few limited elements is adequate for evaluating uncertainties. This paper proposes a new adjoint-based DA methodology that evaluates the uncertainties of estimates by computing limited elements of interest in $\boldsymbol{H}^{-1}$. Owing to this, the proposed method can be applied to simulation models with a huge number of degrees of freedom. We first construct a method to estimate the parameters and initial states involved in an autonomous system, and then validate our approach using a PF model as a testbed. Section II introduces the formulation of an adjoint-based DA method to simultaneously obtain an estimation and its uncertainty using second-order information of the posterior PDF. Section III describes the formulation of an estimation test using synthetic data based on Kobayashi PF model [1]. Section IV presents and discusses the results of estimation tests, and Sec. V concludes this paper by summarizing the results of this study.

\section{METHOD}

\section{A. State-space model and cost function}

DA based on Bayesian statistics starts by defining a statespace model consisting of a system model and an observation model. The system model describes how a state vector evolves over time in accordance with a given simulation model. The state vector contains all time-dependent variables used in the simulation model and sometimes the model parameters.

Suppose an autonomous simulation model is given by $\partial \boldsymbol{z} / \partial t=\boldsymbol{A}(\boldsymbol{z} ; \boldsymbol{a})$, where $\boldsymbol{z}(t) \in \mathbb{R}^{N_{z}}$ denotes a time-dependent variable and $\boldsymbol{A}: \mathbb{R}^{N_{z}} \rightarrow \mathbb{R}^{N_{z}}$ is a function of $\boldsymbol{z}$ and a time-invariant parameter vector $\boldsymbol{a} \in \mathbb{R}^{N_{a}}$. The system model describes the time evolution of a state vector consisting of $z$ and $\boldsymbol{a}$. Let $\boldsymbol{X}(t)=\left(\boldsymbol{z}^{\top}, \boldsymbol{a}^{\top}\right)^{\top} \in \mathbb{R}^{N}$ be the state vector, where - ${ }^{\top}$ denotes the transpose of $\bullet$ and $N=N_{z}+N_{a}$. Since $\boldsymbol{a}$ is time-invariant, i.e., $\partial \boldsymbol{a} / \partial t=\mathbf{0}$, the system model can be represented by

$$
\frac{\partial \boldsymbol{X}}{\partial t}=\boldsymbol{f}(\boldsymbol{X})
$$

where $\boldsymbol{f}: \mathbb{R}^{N} \rightarrow \mathbb{R}^{N}$ is defined as $f_{i}=A_{i}$ for $1 \leqslant i \leqslant N_{z}$ and $f_{i}=0$ for $N_{z}+1 \leqslant i \leqslant N$.

The observation model describes how $\boldsymbol{X}(t)$ relates to a time series of observation data $\boldsymbol{D}(t) \in \mathbb{R}^{K}$, where $K$ denotes the dimension of the observations. Considering that the data include noise, the observation model can be described as

$$
D=\check{h}(X)+\Omega,
$$

where $\check{\boldsymbol{h}}: \mathbb{R}^{N} \rightarrow \mathbb{R}^{K}$ is an observation operator that outputs quantities from $\boldsymbol{X}$ comparable with the data and $\boldsymbol{\Omega}(t)$ denotes observation noise. In this paper, we assume that $\boldsymbol{f}$ and $\breve{h}$ are nonlinear functions and $\boldsymbol{\Omega}$ is white noise that follows a normal distribution with a diagonal covariance matrix. Our purpose is to obtain the optimum initial state $\boldsymbol{X}(0)$ together with the uncertainties of the variables of interest.

In consideration of PF models, we also assume that $\boldsymbol{X}(0)$ is constrained by

$$
X_{i}^{\text {Lower }}<X_{i}(0)<X_{i}^{\text {Upper }} \quad(i=1, \ldots, N),
$$

where $\boldsymbol{X}^{\text {Lower }} \in \mathbb{R}^{N}$ and $\boldsymbol{X}^{\text {Upper }} \in \mathbb{R}^{N}$ denote the lower and upper bounds of $\boldsymbol{X}(0)$, respectively.

To simplify our formulation, we normalize $\boldsymbol{X}$ as

$$
\theta_{i}(t)=\frac{X_{i}(t)-X_{i}^{\text {Lower }}}{X_{i}^{\text {Upper }}-X_{i}^{\text {Lower }}} \quad(i=1, \ldots, N) .
$$

This leads to the following $\boldsymbol{\theta}$-dependent forms of Eqs. (1)-(3):

$$
\begin{gathered}
\frac{\partial \boldsymbol{\theta}}{\partial t}=\boldsymbol{F}(\boldsymbol{\theta}), \\
\boldsymbol{D}=\boldsymbol{h}(\boldsymbol{\theta})+\boldsymbol{\Omega}, \\
0<\Theta_{i}<1 \quad(i=1, \ldots, N),
\end{gathered}
$$

where $F_{i}(\boldsymbol{\theta})=f_{i}(\boldsymbol{X}) /\left(X_{i}^{\text {Upper }}-X_{i}^{\text {Lower }}\right), \boldsymbol{h}(\boldsymbol{\theta})$ is an observation operator after transforming $\boldsymbol{X}$ to $\boldsymbol{\theta}$, and $\Theta=\boldsymbol{\theta}(0)$. 
Bayes' theorem states that a conditional PDF $p(\boldsymbol{\Theta} \mid \boldsymbol{D})$, which is called the posterior PDF, can be described as

$$
p(\boldsymbol{\Theta} \mid \boldsymbol{D})=\frac{p(\boldsymbol{\Theta}) p(\boldsymbol{D} \mid \boldsymbol{\Theta})}{p(\boldsymbol{D})},
$$

where $p(\boldsymbol{\Theta})$ and $p(\boldsymbol{D} \mid \boldsymbol{\Theta})$ are called the prior PDF and likelihood, respectively. Note that $p(\boldsymbol{D})$ is constant, since $\boldsymbol{D}$ is a definite vector. Thus, Eq. (8) implies that $p(\boldsymbol{\Theta} \mid \boldsymbol{D})$ is proportional to a product of the prior PDF and the likelihood.

The prior PDF contains prior information provided by experience and intuition. If we suppose that this prior information is the constraint condition given by Eq. (7) and that $\Theta_{i}$ is independent for each $i$, the joint PDF $p(\boldsymbol{\Theta})$ is given by a product of prior PDFs of $\Theta_{i}$,

$$
p(\Theta)=\prod_{i=1}^{N} p\left(\Theta_{i}\right),
$$

where

$$
p\left(\Theta_{i}\right)= \begin{cases}1 & \left(0<\Theta_{i}<1\right) \\ 0 & \text { (otherwise) } .\end{cases}
$$

When observation data are obtained at $t=t_{1}, t_{2}, \ldots$, $t_{n}, p(\boldsymbol{D} \mid \boldsymbol{\Theta})$ can be written as

$$
p(\boldsymbol{D} \mid \boldsymbol{\Theta})=\prod_{s=1}^{n} \prod_{k=1}^{K} p\left(\Omega_{k}\left(t_{s}\right)\right),
$$

where

$$
p\left(\Omega_{k}\left(t_{s}\right)\right)=\frac{1}{\sqrt{2 \pi \sigma_{k}^{2}}} \exp \left[-\frac{\left\{D_{k}\left(t_{s}\right)-h_{k}\left(\boldsymbol{\theta}\left(t_{s}\right)\right)\right\}^{2}}{2 \sigma_{k}^{2}}\right],
$$

and $\sigma_{k}$ is the standard deviation of $\Omega_{k}(k=1, \ldots, K)$. We consider $\sigma_{k}$ to be a hyper-parameter.

This paper defines the optimum solution $\hat{\boldsymbol{\Theta}}$ to be the $\boldsymbol{\Theta}$ that maximizes the posterior PDF $p(\boldsymbol{\Theta} \mid \boldsymbol{D})$. For the convenience of numerical computation, we aim to minimize a cost function

$$
\begin{gathered}
J=\sum_{s=1}^{n} \sum_{k=1}^{K}\left[\frac{\log \left(2 \pi \sigma_{k}^{2}\right)}{2}+\frac{\left\{D_{k}\left(t_{s}\right)-h_{k}\left(\boldsymbol{\theta}\left(t_{s}\right)\right)\right\}^{2}}{2 \sigma_{k}^{2}}\right] \\
\text { subject to } 0<\Theta_{i}<1,
\end{gathered}
$$

which comes from a negative logarithmic posterior PDF, i.e., $p(\boldsymbol{\Theta} \mid \boldsymbol{D}) \propto e^{-J}$, to find $\hat{\boldsymbol{\Theta}}$, rather than maximizing $p(\boldsymbol{\Theta} \mid \boldsymbol{D})$. The constraint in Eq. (13) arises from the term $-\log p(\boldsymbol{\Theta})$, which appears when calculating $-\log p(\boldsymbol{\Theta} \mid \boldsymbol{D})$.

An optimum solution $\hat{\sigma}_{k}$ for $\sigma_{k}$ can be determined as follows. By letting $\partial J / \partial \sigma_{k}=0$, we obtain

$$
\sigma_{k}=\sqrt{\frac{1}{n} \sum_{s=1}^{n}\left\{D_{k}\left(t_{s}\right)-h_{k}\left(\boldsymbol{\theta}\left(t_{s}\right)\right)\right\}^{2}} .
$$

Then, $\hat{\sigma}_{k}$ is obtained by substituting $\boldsymbol{\theta}$, which is calculated by Eq. (5) using $\boldsymbol{\theta}(0)=\hat{\boldsymbol{\Theta}}$, into Eq. (14).

\section{B. Optimization via an adjoint method}

Typically, $J$ is optimized using a gradient method such as the steepest gradient descent, the nonlinear conjugate gradient method, or the limited-memory Broyden-Fletcher-GoldfarbShanno (LBFGS) method [35]. Gradient methods require $\partial J /$ $\partial \boldsymbol{\Theta}$ to update $J$, but it is difficult to calculate this quantity because $J$ does not explicitly include $\boldsymbol{\Theta}$, as seen in Eq. (13). Generally, $J$ is fully determined by setting $\Theta=\boldsymbol{\theta}(0)$ through Eq. (5), so that $J$ must be written as a function of $\boldsymbol{\Theta}$, i.e., $J(\Theta)$. According to Eq. (13), $J$ is also a function of $\boldsymbol{\theta}\left(t_{s}\right)(s=$ $1, \ldots, n)$ that satisfies Eq. (5), not including $\Theta$. Summarizing these two expressions for $J$ :

$$
J(\boldsymbol{\Theta})=\int_{0}^{t_{f}} d t \mathcal{J}(\boldsymbol{\theta})+\int_{0}^{t_{f}} d t \lambda^{\top}\left(\boldsymbol{F}-\frac{\partial \boldsymbol{\theta}}{\partial t}\right),
$$

where $t_{f}$ is an arbitrary time later than $t_{n}$, and $\lambda(t) \in \mathbb{R}^{N}$ denotes a vector of the Lagrange multipliers that impose Eq. (5) as the constraint condition of $\boldsymbol{\theta}$. $\mathcal{J}$ is the time-dependent function

$\mathcal{J}(\boldsymbol{\theta})=\sum_{s=1}^{n} \delta\left(t-t_{s}\right) \sum_{k=1}^{K}\left[\frac{\log \left(2 \pi \sigma_{k}^{2}\right)}{2}+\frac{\left\{D_{k}(t)-h_{k}(\boldsymbol{\theta}(t))\right\}^{2}}{2 \sigma_{k}^{2}}\right]$,

which satisfies $J=\int_{0}^{t_{f}} d t \mathcal{J}$, where $\delta(t)$ denotes the Dirac delta function. Taking a variation of Eq. (15), we have a time evolution equation for $\lambda$ :

$$
\frac{\partial \boldsymbol{\lambda}}{\partial t}+\left(\frac{\partial \boldsymbol{F}}{\partial \boldsymbol{\theta}}\right)^{\top} \lambda+\frac{\partial \mathcal{J}}{\partial \boldsymbol{\theta}}=\mathbf{0},
$$

where

$$
\begin{gathered}
\lambda(0)=\frac{\partial J}{\partial \boldsymbol{\Theta}}, \\
\lambda\left(t_{f}\right)=\mathbf{0} .
\end{gathered}
$$

Details of the derivation can be found in Refs. [26,36]. Solving Eq. (17) backwardly in time with the condition in Eq. (19), we obtain the objective $\partial J / \partial \Theta$ as $\lambda(0)$. Such a procedure to obtain the gradient of the cost function using the adjoint equation [Eq. (17)] is called the adjoint method.

When we apply the adjoint method to our problem, a variable transformation is needed in the process of updating $\Theta$ based on a gradient method, since $\Theta$ has the constraint shown in Eq. (7). The variable transformation,

$$
\Psi_{i}=\log \Theta_{i}-\log \left(1-\Theta_{i}\right) \quad(i=1, \ldots, N),
$$

converts the constrained optimization problem of $\Theta$ into an unconstrained one with respect to $\boldsymbol{\Psi}$. The update procedure is as follows. After obtaining $\partial J / \partial \Theta$ by the adjoint method based on Eqs. (17)-(19), we convert $\boldsymbol{\Theta}$ to $\boldsymbol{\Psi}$ using Eq. (20) and $\partial J / \partial \boldsymbol{\Theta}$ to $\partial J / \partial \boldsymbol{\Psi}$ as

$$
\frac{\partial J}{\partial \Psi_{i}}=\Theta_{i}\left(1-\Theta_{i}\right) \frac{\partial J}{\partial \Theta_{i}} \quad(i=1, \ldots, N) .
$$

Using this formulation to update $\Psi$, we can obtain an updated $\Theta$ from the inverse transformation of Eq. (20):

$$
\Theta_{i}=\frac{1}{1+\exp \left(-\Psi_{i}\right)} \quad(i=1, \ldots, N) .
$$

Although this update procedure does not allow $\Theta_{i}$ to be exactly 0 or 1, owing to the definition of Eq. (22), $\Theta_{i}$ can be sufficiently close to 0 or 1 , which poses no problem in practical cases. 
The adjoint method calculates $\partial J / \partial \Theta$ for a fixed $\sigma_{k}$, so that an optimization of $\sigma_{k}$ is to be done at the same time as $\boldsymbol{\Theta}$ by substituting Eq. (14) into Eq. (16) every time $\boldsymbol{\Theta}$ is updated.

The advantages of the adjoint method over sequential Bayesian filters are that only $O(C)$ computations and $O(N)$ memory are required to find $\hat{\boldsymbol{\Theta}}$, where $C$ is the number of computations needed to run the given simulation model from $t=0$ to $t=t_{f}$.

\section{Evaluation of uncertainty via a second-order adjoint method}

The adjoint method described in Sec. II B gives the optimum solution $\hat{\boldsymbol{\Theta}}$ that maximizes $p(\boldsymbol{\Theta} \mid \boldsymbol{D})$. However, it does not provide information about the behavior of $p(\boldsymbol{\Theta} \mid \boldsymbol{D})$ in the neighborhood of $\boldsymbol{\Theta}=\hat{\boldsymbol{\Theta}}$, which reflects the uncertainty in the estimation of $\hat{\boldsymbol{\Theta}}$. To extract such information, another procedure must be implemented on the adjoint method. Considering that $\partial J /\left.\partial \boldsymbol{\Theta}\right|_{\boldsymbol{\Theta}=\hat{\boldsymbol{\Theta}}}=\mathbf{0}$, the Taylor expansion of $J$ with respect to $\boldsymbol{\Theta}-\hat{\boldsymbol{\Theta}}$ is

$$
J(\boldsymbol{\Theta}) \sim J(\hat{\boldsymbol{\Theta}})+\frac{1}{2}(\boldsymbol{\Theta}-\hat{\boldsymbol{\Theta}})^{\top} \boldsymbol{H}(\boldsymbol{\Theta}-\hat{\boldsymbol{\Theta}}),
$$

where terms of order higher than three have been neglected, and $\boldsymbol{H}$ is a Hessian matrix given by

$$
H_{i, j}=\left.\frac{\partial^{2} J}{\partial \Theta_{i} \partial \Theta_{j}}\right|_{\boldsymbol{\Theta}=\hat{\boldsymbol{\Theta}}} \quad(i, j=1, \ldots, N) .
$$

We normalize $p(\boldsymbol{\Theta} \mid \boldsymbol{D}) \propto e^{-J}$ into which Eq. (23) is substituted as

$$
p(\boldsymbol{\Theta} \mid \boldsymbol{D}) \sim \frac{\exp \left[-\frac{1}{2}(\boldsymbol{\Theta}-\hat{\boldsymbol{\Theta}})^{\top} \boldsymbol{H}(\boldsymbol{\Theta}-\hat{\boldsymbol{\Theta}})\right]}{(2 \pi)^{N / 2}\left|\boldsymbol{H}^{-1}\right|^{1 / 2}},
$$

where $\boldsymbol{H}^{-1}$ is the inverse of $\boldsymbol{H}$ and $|\bullet|$ denotes the determinant of •. Equation (25) indicates that, in the neighborhood of $\boldsymbol{\Theta}=\hat{\boldsymbol{\Theta}}, p(\boldsymbol{\Theta} \mid \boldsymbol{D})$ can be approximated by a multivariate normal distribution with mean vector $\hat{\boldsymbol{\Theta}}$ and covariance matrix $\boldsymbol{H}^{-1}$. Let $\Theta_{l}(1 \leqslant l \leqslant N)$ be a component of interest in $\boldsymbol{\Theta}$. Integrating Eq. (25) over all variables except for $\Theta_{l}$, the marginal distribution with respect to $\Theta_{l}$ is the normal distribution with mean $\hat{\Theta}_{l}$ and variance $\left(H^{-1}\right)_{l, l}$, which is the $l$ th diagonal element of $\boldsymbol{H}^{-1}$. This means that the uncertainty of $\Theta_{l}$ is given by $\left(H^{-1}\right)_{l, l}$. When $N \gg 1$, it is unrealistic to obtain $\boldsymbol{H}^{-1}$ directly, as this would require $O\left(C N^{2}+N^{3}\right)$ computations and $O\left(N^{2}\right)$ memory. In practical cases, it is not necessary to evaluate all elements of $\boldsymbol{H}^{-1}$, since the number of elements of interest is usually much smaller than $N$.

We propose a new methodology based on a second-order adjoint method $[37,38]$ to efficiently obtain such uncertainties in massive autonomous systems. The following procedure to obtain the uncertainties requires $O(C)$ computations and $O(N)$ memory for each uncertainty. When evaluating the uncertainty of $\Theta_{l}$, we consider a linear equation of $\boldsymbol{r} \in \mathbb{R}^{N}$ :

$$
\boldsymbol{H r}=\boldsymbol{q},
$$

where $\boldsymbol{q} \in \mathbb{R}^{N}$ is a vector with elements $q_{l}=1$ and $q_{i \neq l}=$ 0 . The solution $\hat{\boldsymbol{r}}$ obviously includes $\left(H^{-1}\right)_{l, l}$, since $\hat{r}_{l}=$ $\sum_{j=1}^{N}\left(H^{-1}\right)_{l, j} q_{j}=\left(H^{-1}\right)_{l, l}$. Note that $\boldsymbol{H}$ is a constant matrix that requires complex computations because of its large dimension. We must obtain $\hat{\boldsymbol{r}}$ from an initial guess $\boldsymbol{r}_{0}$ via an iterative technique such as the conjugate gradient method or the conjugate residual method. The iterative method needs, in the way of the iteration, to compute many Hessian-vector products $\boldsymbol{H} \boldsymbol{r}_{k}$, where $\boldsymbol{r}_{k}$ is the $k$ th update. The second-order adjoint method enables us to compute such Hessian-vector products.

Let $\boldsymbol{\xi}(t) \in \mathbb{R}^{N}$ and $\zeta(t) \in \mathbb{R}^{N}$ be perturbations of $\hat{\boldsymbol{\theta}}$ and $\hat{\lambda}$, which correspond to $\boldsymbol{\theta}$ and $\lambda$, respectively, when $\boldsymbol{\Theta}=\hat{\boldsymbol{\Theta}}$. Their time evolutions are given by

$$
\begin{gathered}
\frac{\partial \boldsymbol{\xi}}{\partial t}=\left.\frac{\partial \boldsymbol{F}}{\partial \boldsymbol{\theta}}\right|_{\boldsymbol{\theta}=\hat{\boldsymbol{\theta}}} \boldsymbol{\xi} \\
\frac{\partial \boldsymbol{\zeta}}{\partial t}+\left.\left(\frac{\partial \boldsymbol{F}}{\partial \boldsymbol{\theta}}\right)^{\top}\right|_{\boldsymbol{\theta}=\hat{\boldsymbol{\theta}}} \boldsymbol{\zeta}+\left.\left(\frac{\partial^{2} \boldsymbol{F}}{\partial \boldsymbol{\theta}^{2}} \boldsymbol{\xi}\right)^{\top}\right|_{\boldsymbol{\theta}=\hat{\boldsymbol{\theta}}} \hat{\lambda}+\left.\frac{\partial^{2} \mathcal{J}}{\partial \boldsymbol{\theta}^{2}}\right|_{\boldsymbol{\theta}=\hat{\boldsymbol{\theta}}} \boldsymbol{\xi}=\mathbf{0}
\end{gathered}
$$

The combination of Eqs. (27) and (28), which are called the tangent linear model and second-order adjoint model, respectively, gives the Hessian-vector product for an arbitrary vector. Solving Eq. (27) forwardly in time for a given vector $\boldsymbol{\xi}(0)=\boldsymbol{\gamma}$, we obtain the time series of $\boldsymbol{\xi}$. Then, solving Eq. (28) backwardly with the given $\zeta\left(t_{f}\right)=\mathbf{0}$ and the time series $\boldsymbol{\xi}$, we obtain the objective Hessian-vector product $\zeta(0)=\boldsymbol{H} \boldsymbol{\gamma}$. The detailed derivation is given in Ref. [37].

We now discuss the computational cost of solving Eq. (26) by some iterative technique, e.g., the conjugate gradient method or the conjugate residual method, together with the second-order adjoint method. First, obtaining an objective Hessian-vector product for a given vector requires $O(C)$ computations when solving Eqs. (27) and (28). Then, updating the vector in each iteration needs $O(N)$ computations. Therefore, the number of all computations in one iteration is $O(C)+$ $O(N)=O(C)$, since $C \geqslant N$. Consequently, obtaining the solution requires $O(C)$ computations, considering that the number of iterations needed for convergence does not depend on $N$.

\section{TWIN EXPERIMENT}

\section{A. Kobayashi phase-field model}

The accuracy of the proposed method is verified through numerical simulations termed "twin experiments," details of which are given in Sec. III B. We choose a two-dimensional Kobayashi PF model as the testbed in the twin experiments. Kobayashi PF model describes the fundamental growth dynamics of two phases, such as in solidification or a phase transformation. The time evolution of one of the phases is described by

$$
\tau \frac{\partial \phi}{\partial t}=\epsilon^{2} \triangle \phi+\phi(1-\phi)\left(\phi+m-\frac{1}{2}\right),-\frac{1}{2}<m<\frac{1}{2},
$$

where the PF variable $\phi(\boldsymbol{x}, t)$ denotes the existence probability of the relevant phase, e.g., solid or liquid. The parameters $\tau$ and $\epsilon$ nondimensionalize time and space, respectively, and $m$ characterizes the velocity of the interface between the two phases. We assume that these parameters are time-invariant constants. We know that $\phi(x, t)$ should be constrained in $0 \leqslant \phi(\boldsymbol{x}, t) \leqslant 1$, as it describes a probability. This condition is automatically satisfied by setting the initial phase to $0 \leqslant$ 
$\phi(x, 0) \leqslant 1$, since $\phi=0$ and $\phi=1$ are the fixed points of Eq. (29).

Kobayashi PF model underlies various PF models that describe physical phenomena such as dendrite growth [1,5], crack propagation $[39,40]$, and interface-driven pattern formation [41]. Therefore, Kobayashi PF model is a good choice for verifying whether the proposed DA method works well and is a first step towards future applications in more complex PF models.

\section{B. Synthetic data}

Twin experiments are often conducted in the field of DA to verify a newly developed method on the basis of synthetic data. The synthetic data are usually generated using the given simulation model, in which the true parameters and initial state are predetermined. Verification then proceeds by checking whether the DA method applied to the synthetic data reproduces the true parameters and initial state. In our case, the synthetic dataset is a time series of $\phi$ that is numerically calculated by Kobayashi PF model with a true initial state and parameter $m$. The synthetic data are then contaminated by observation noise that follows a normal distribution with mean zero and variance $\sigma^{2}$. The twin experiments are intended to confirm that the proposed method estimates the initial state and parameter with the associated uncertainty.

Let $n_{x}$ and $n_{y}$ be the numbers of grid points in the $x$ - and $y$-directions, respectively, $M$ be the total number of grid points, i.e., $M=n_{x} n_{y}$, and $h$ be the grid spacing. A periodic boundary condition is imposed on the boundary of the computational domain. Letting $\phi_{i}(t)$ be the phase at the $i$ th grid point, Eq. (29) can be rewritten as

$$
\tau \frac{\partial \phi_{i}}{\partial t}=\epsilon^{2} \triangle_{i} \phi_{i}+\phi_{i}\left(1-\phi_{i}\right)\left(\phi_{i}+m-\frac{1}{2}\right),
$$

where $\triangle_{i}$ denotes a second-order difference operator acting on the four nearest neighbors of the $i$ th grid point $\mathcal{S}_{i}$; i.e., $\triangle_{i} \phi_{i}=\sum_{j \in \mathcal{S}_{i}}\left(\phi_{j}-\phi_{i}\right) / h^{2}$.

Figure 1 shows the time evolution of $\phi$ in two-dimensional space, where $n_{x}=300, n_{y}=200, h=\epsilon$, and the time increment in the Euler method is $0.1 \tau$. The assumed initial state is shown in Fig. 1(a), and the true value for the parameter $m$ is assumed to be 0.1 . Figures 1(b)-1(f) show snapshots
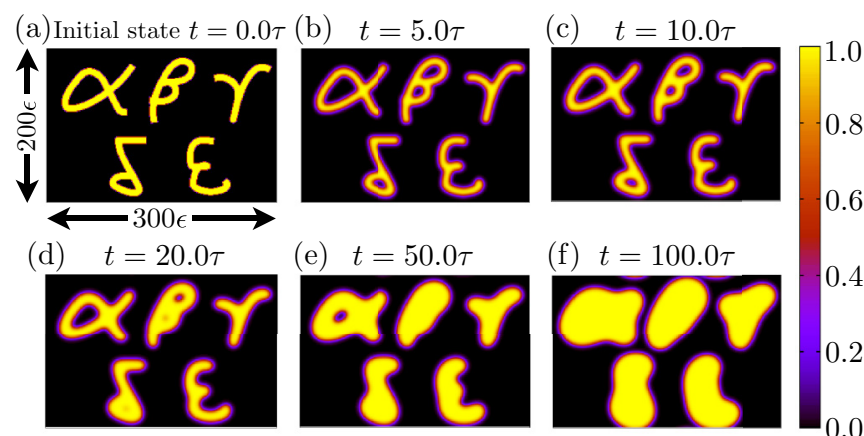

(e) $t=50.0 \tau$

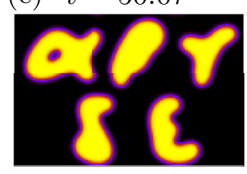

(f) $t=100.0 \tau$

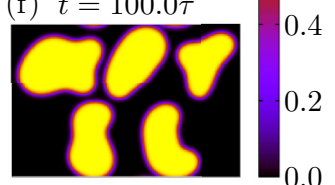

FIG. 1. Time evolution of phase field $\phi$ starting from the initial state shown in (a) for $m=0.1$. (b)-(f) $\phi$ at $t=$ $5.0 \tau, 10.0 \tau, 20.0 \tau, 50.0 \tau$, and $100.0 \tau$, respectively. The color indicates the magnitude of $\phi$. indicating that the interface between the phases $\phi=0$ and $\phi=1$ migrates, expanding the area of $\phi=1$. Motivated by the fact that such snapshots are sometimes obtained as observation data in practical experiments, we use snapshots such as in Figs. 1(b)-1(f) with added observation noise as the synthetic data for the twin experiments. The synthetic data are given by

$$
\phi_{i}^{\mathrm{obs}}(t)=\phi_{i}(t)+\omega_{i}(t) \quad(i=1, \ldots, M),
$$

where $\omega_{i}(t)$ is normally distributed observation noise with mean zero and variance $\sigma^{2}$.

When DA is applied to Kobayashi PF model, the time evolution equation with respect to $m$ is needed to construct a system model within the state-space model (Sec. II A). The time evolution equation can be written as

$$
\tau \frac{\partial b}{\partial t}=0
$$

where $b=m+1 / 2$, which denotes the normalization of $m$, i.e., $0<b<1$.

\section{Cost function}

We consider the synthetic observation data to be the snapshots of $\phi$ obtained from $t=T_{\min }$ to $t=T_{\max }$ with time interval $\Delta T$. Let $\mathcal{T}$ be the set of observation times and $n$ be the number of observations. Combining Eqs. (13) and (31), $J$ can be rewritten as

$$
J=\frac{n M}{2} \log \left(2 \pi \sigma^{2}\right)+\frac{1}{2 \sigma^{2}} \sum_{t_{s} \in \mathcal{T}} \sum_{i=1}^{M}\left[\phi_{i}^{\mathrm{obs}}\left(t_{s}\right)-\phi_{i}\left(t_{s}\right)\right]^{2} .
$$

The values of $\phi_{i}(0)$ and $b(0)$ that minimize Eq. (33) also minimize

$$
J^{\prime}=\int_{0}^{T} d t \mathcal{J}^{\prime}
$$

where

$$
\mathcal{J}^{\prime}=\frac{1}{2} \sum_{t_{s} \in \mathcal{T}} \delta\left(t-t_{s}\right) \sum_{i=1}^{M}\left[\phi_{i}^{\mathrm{obs}}(t)-\phi_{i}(t)\right]^{2},
$$

because $\sigma$ is independent of $\phi_{i}(0)$ and $b(0)$. When the optimum $\hat{\phi}_{i}(0)$ for $\phi_{i}(0)$ and $\hat{b}(0)$ for $b(0)$ are obtained by minimizing $J^{\prime}$, the optimum $\sigma$ can be obtained as

$$
\hat{\sigma}=\sqrt{\frac{1}{n M} \sum_{t_{s} \in \mathcal{T}} \sum_{i=1}^{M}\left[\phi_{i}^{\mathrm{obs}}\left(t_{s}\right)-\hat{\phi}_{i}\left(t_{s}\right)\right]^{2}},
$$

where $\hat{\phi}_{i}(t)$ denotes $\phi_{i}(t)$ simulated using $\hat{\phi}_{i}(0)$ and $\hat{b}(0)$.

\section{Procedures}

Prior to applying the proposed method to the PF model, the constraint for the initial state $0 \leqslant \phi_{i}(0) \leqslant 1$ is changed to $0<$ $\phi_{i}(0)<1$ to satisfy the domain of the variable transformation Eq. (20). The state variables $\boldsymbol{\theta}(t) \in \mathbb{R}^{M+1}$ and $\boldsymbol{\Theta} \in \mathbb{R}^{M+1}$ can be defined as

$$
\begin{aligned}
\boldsymbol{\theta} & =\left(\phi_{1}, \ldots, \phi_{M}, b\right)^{\top}, \\
\boldsymbol{\Theta} & =\left(\phi_{1}(0), \ldots, \phi_{M}(0), b(0)\right)^{\top} .
\end{aligned}
$$


The constraint for $\Theta$ becomes $0<\Theta_{i}<1(i=1, \ldots, M+1)$. The system models of Eqs. (30) and (32) are rewritten in terms of $\boldsymbol{\theta}$ as

$$
\tau \frac{\partial \theta_{i}}{\partial t}=\left\{\begin{array}{l}
\epsilon^{2} \triangle_{i} \theta_{i}+\theta_{i}\left(1-\theta_{i}\right)\left(\theta_{i}+\theta_{M+1}-1\right) \quad(i=1, \ldots, M), \\
0 \quad \text { (otherwise) }
\end{array}\right.
$$

Substituting the right-hand side of this equation for $\boldsymbol{F}$ in Eq. (17), replacing $\mathcal{J}$ [Eq. (17)] with $\mathcal{J}^{\prime}$ [Eq. (35)], and replacing $J$ [Eq. (18)] with $J^{\prime}$ [Eq. (34)], the adjoint method described by Eqs. (17)-(19) is rewritten as

$$
-\tau \frac{\partial \lambda_{i}}{\partial t}=\left\{\begin{array}{cc}
\epsilon^{2} \triangle_{i} \lambda_{i}+\left\{-3 \theta_{i}^{2}+\left(4-2 \theta_{M+1}\right) \theta_{i}+\theta_{M+1}-1\right\} \lambda_{i}+\frac{\partial \mathcal{J}^{\prime}}{\partial \theta_{i}} \quad(i=1, \ldots, M), \\
\sum_{j=1}^{M} \theta_{j}\left(1-\theta_{j}\right) \lambda_{j} \quad \text { (otherwise) }, \\
\lambda(0)=\frac{\partial J^{\prime}}{\partial \boldsymbol{\Theta}} \\
\lambda\left(t_{f}\right)=\mathbf{0} .
\end{array}\right.
$$

We adopt the LBFGS technique [35] as the gradient method for optimizing $\Theta$. Starting from an initial guess, the LBFGS method updates $\Theta$ by satisfying $0<\Theta_{i}<1$ for all $i$ owing to the variable transformation mentioned in Sec. II B. To tune the LBFGS method, we set the tolerance to $10^{-8}$ and determine the step length by Armijo's rule [42]. Once the optimum $\hat{\Theta}$ has been obtained, the optimum standard deviation $\hat{\sigma}$ can be estimated by Eq. (36) and the optimum $\hat{m}$ for $m$ is given by $\hat{\Theta}_{M+1}-1 / 2$ or $\hat{b}(0)-1 / 2$.

One of the most remarkable features of the proposed method is its evaluation of the uncertainties. These uncertainties can provide important information that is beneficial to updating the experimental design. In accordance with the procedure mentioned in Sec. II C, we consider a linear equation $\boldsymbol{H}^{\prime} \boldsymbol{r}=\boldsymbol{q}$, where $\boldsymbol{H}^{\prime}=\partial^{2} \boldsymbol{J}^{\prime} /\left.\partial \boldsymbol{\Theta}^{2}\right|_{\boldsymbol{\Theta}=\boldsymbol{\Theta}}$ is a Hessian matrix, $\boldsymbol{r} \in \mathbb{R}^{M+1}$ is a vector to be determined, and $\boldsymbol{q} \in \mathbb{R}^{M+1}$ is a vector containing the elements $q_{M+1}=1$ and $q_{i \neq M+1}=0$. The uncertainty $\delta \hat{m}$ can be computed from the solution $\hat{\boldsymbol{r}}$ as

$$
\delta \hat{m}=\hat{\sigma} \sqrt{\hat{r}_{M+1}} .
$$

The conjugate residual method, in which the tolerance is set to $10^{-8}$, is adopted to solve the linear equation. The second-order adjoint method computes each of the Hessian-vector products $\boldsymbol{H}^{\prime} \boldsymbol{\gamma}$, where $\boldsymbol{\gamma}$ indicates an arbitrary vector, that appear in the optimization process of the conjugate residual method. Substituting the right-hand side of Eq. (38) for $\boldsymbol{F}$ in Eq. (27), the tangent linear model can be rewritten as

$$
\tau \frac{\partial \xi_{i}}{\partial t}=\left\{\begin{array}{l}
\epsilon^{2} \triangle_{i} \xi_{i}+\hat{\theta}_{i}\left(1-\hat{\theta}_{i}\right) \xi_{M+1}+\left\{-3 \hat{\theta}_{i}^{2}+\left(4-2 \hat{\theta}_{M+1}\right) \hat{\theta}_{i}+\hat{\theta}_{M+1}-1\right\} \xi_{i} \quad(i=1, \ldots, M), \\
0 \quad \text { (otherwise), }
\end{array}\right.
$$

with the initial condition $\boldsymbol{\xi}(0)=\boldsymbol{\gamma}$, where $\hat{\boldsymbol{\theta}}$ denotes the state vector corresponding to $\hat{\boldsymbol{\Theta}}$. Substituting the right-hand side of Eq. (38) for $\boldsymbol{F}$ in Eq. (28) and replacing $\mathcal{J}$ [Eq. (28)] with $\mathcal{J}^{\prime}$ [Eq. (35)], the second-order adjoint model in Eq. (28) becomes

$$
-\tau \frac{\partial \zeta_{i}}{\partial t}=\left\{\begin{array}{l}
\epsilon^{2} \triangle_{i} \zeta_{i}+\left\{-3 \hat{\theta}_{i}^{2}+\left(4-2 \hat{\theta}_{M+1}\right) \hat{\theta}_{i}+\hat{\theta}_{M+1}-1\right\} \zeta_{i} \\
-\left(6 \hat{\theta}_{i}+2 \hat{\theta}_{M+1}-4\right) \hat{\lambda}_{i} \xi_{i}-\left(2 \hat{\theta}_{i}-1\right) \hat{\lambda}_{i} \xi_{M+1}+\left.\sum_{j=1}^{M} \frac{\partial^{2} \mathcal{J}^{\prime}}{\partial \theta_{i} \partial \theta_{j}}\right|_{\theta=\hat{\theta}_{j}} \quad(i=1, \ldots, M), \\
\sum_{j=1}^{M}\left[\hat{\theta}_{j}\left(1-\hat{\theta}_{j}\right) \zeta_{j}-\left(2 \hat{\theta}_{j}-1\right) \hat{\lambda}_{j} \xi_{j}\right] \quad \text { (otherwise), }
\end{array}\right.
$$

where $\hat{\lambda}$ is the perturbation of $\hat{\boldsymbol{\theta}}$. Solving Eq. (44) with the condition $\zeta\left(t_{f}\right)=\mathbf{0}$, we obtain the objective Hessian-vector product as $\zeta(0)=\boldsymbol{H}^{\prime} \boldsymbol{\gamma}$.

\section{RESULTS AND DISCUSSION}

The proposed method is verified through three twin experiments: (I) estimation of the parameter $m$ conditional on the true initial state $\phi_{i}^{\text {true }}(0)$ (Sec. IV A), (II) simultaneous estimation of the parameter $m$ and the initial state $\phi_{i}(0)$ (Sec. IV B), and (III) estimation of the initial state $\phi_{i}(0)$ conditional on the true parameter $m^{\text {true }}$ (Sec. IV C). Twin experiment I investigates how the estimation depends on observation data, twin experi- ment II verifies whether the proposed method outputs correct estimations, even for massive simulation models, and twin experiment III validates the unknown phenomena that appear in the results of experiment II.

\section{A. Twin experiment I: Parameter estimation}

Twin experiment I investigates the influences of three parameters related to the observation data: (i) the length of the observation time $T_{\max }$, (ii) the time interval of the observations $\Delta T$, and (iii) the standard deviation of the observation noise $\sigma$. Table I summarizes the parameter values used in the experiments. The first observation is assumed to 
TABLE I. Length of the observation time $T_{\max }$, time interval of observations $\Delta T$, and standard deviation of observation noise $\sigma$ used in twin experiment I, where $\tau$ is the unit of time in the simulation. Test I-(i), -(ii), and -(iii) investigate how the estimation depends on $T_{\max }, \Delta T$, and $\sigma$, respectively.

\begin{tabular}{lccc}
\hline \hline & $T_{\max }$ & $\Delta T$ & $\sigma$ \\
\hline Test I-(i) & $0.2 \tau-102.4 \tau$ & $0.1 \tau$ & 0.01 \\
Test I-(ii) & $102.5 \tau$ & $0.1 \tau-51.2 \tau$ & 0.01 \\
Test I-(iii) & $102.4 \tau$ & $0.1 \tau$ & $10^{-5}-1.0$ \\
\hline \hline
\end{tabular}

occur at $T_{\min }=0.1 \tau$, and the true value of $m$ is assumed to be $m^{\text {true }}=0.1$. The initial guess used in the LBFGS method is $m=-0.1$. In this experiment, the true phase field at $t=0$, i.e., $\phi_{i}^{\text {true }}(0)$ [see Fig. 1(a)] is given as the initial state. The results reported here are the average values of $\hat{m}$ and $\delta \hat{m}$ over 20 trials with different random seeds.

Figure 2 shows the results of Test I-(i). Figure 2(a) indicates that the parameter estimation is successful, because the true parameter is included in the range $\hat{m}-\delta \hat{m}<m^{\text {true }}<\hat{m}+\delta \hat{m}$. The estimation of the uncertainty $\delta \hat{m}$ fails when $T_{\max }$ is at its minimum, i.e., $T_{\max }=0.2 \tau$, because insufficient data cause $\hat{r}_{M+1}$ to become negative. Figure 2(b) indicates that $\delta \hat{m}$ is proportional to $T_{\max }^{-1.5}$ in this range. The reason that the decrease is more rapid than the law of large numbers would suggest is related to the nonlinearity of Kobayashi PF model. A theoretical evaluation actually indicates that $\delta \hat{m}$ is proportional to $T_{\max }^{-2.5}$ when $T_{\max } \ll \tau$, and will converge with a constant value as $T_{\max }$ increases. This is because no additional information is included in the observation data after $\phi(x, t)$ becomes almost uniform across the entire computational domain.

Figure 3 shows the results of Test I-(ii). Figure 3(a) indicates that the proposed method successfully reproduces the true parameter, and Fig. 3(b) shows that $\delta \hat{m}$ is proportional to $\Delta T^{0.5}$ when $\Delta T<\tau$, which seems to follow the law of large numbers.

Figure 4 shows the results of Test I-(iii). Figure 4(a) indicates that the parameter estimation is again successful, and Fig. 4(b) shows that $\delta \hat{m}$ is proportional to $\sigma$. In summary, the results of Test I demonstrate that the proposed method is capable of estimating the true parameter and the associated uncertainty.
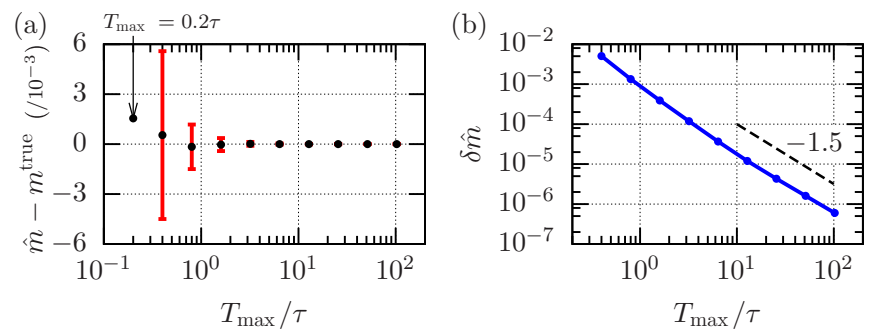

FIG. 2. Results of twin experiment I-(i). The length of each error bar for the optimum parameter $\hat{m}$ in (a) corresponds to the estimated uncertainty $\delta \hat{m}$ in (b). The uncertainty cannot be determined when $T_{\max }=0.2 \tau$. The dashed black line in (b) indicates a power function of order -1.5 .
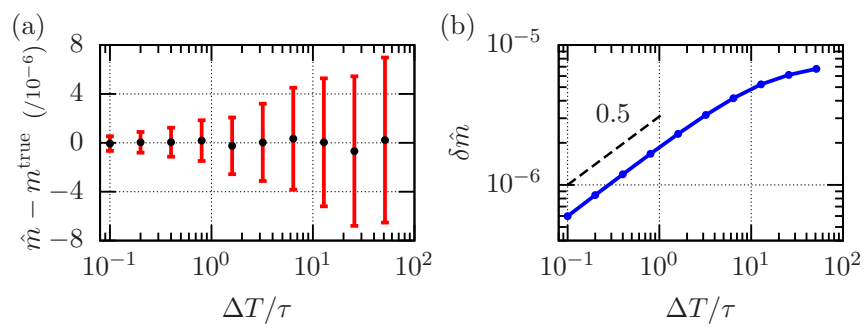

FIG. 3. Results of twin experiment I-(ii). The length of each error bar for optimum parameter $\hat{m}$ in (a) corresponds to the estimated uncertainty $\delta \hat{m}$ in (b). The dashed black line in (b) indicates a power function of order of 0.5 .

\section{B. Twin experiment II: Simultaneous estimation of parameter and initial state}

Twin experiment II investigates the influence of the observation noise in two cases: (i) when the noise has a small standard deviation $\left(\sigma=10^{-4}\right)$ and (ii) when the noise has a large standard deviation $(\sigma=0.3)$. The true parameter is assumed to be $m^{\text {true }}=0.1$, and the true initial state $\phi_{i}^{\text {true }}(0)$ is assumed to be the phase field shown in Fig. 1(a). The other observational conditions are $T_{\min }=5.0 \tau, T_{\max }=30.0 \tau, \Delta T=0.1 \tau$, and the initial guesses are $\phi_{i}(0)=0.2$ and $m=-0.2$.

Figure 5 shows the results of Test II. Figure 5(a) indicates how each iteration of the LBFGS method updates the estimation of $m$. It is clear that each estimation converges with $m^{\text {true }}$. Figures 5(b) and 5(c) indicate the estimated initial states $\hat{\phi}_{i}(0)$ in Test II-(i) and (ii), respectively. These results appear to be almost consistent with the true initial states, although a "spotlike" pattern appears in Fig. 5(c). This spotlike pattern would be conspicuous if the observation noise was large or if the time of the first observation $T_{\min }$ was far from $t=0$. Additionally, the spotlike pattern does not disappear under lower tolerance levels.

\section{Twin experiment III: Estimation of initial state}

Twin experiment III confirms whether the estimation of $m$ affects the generation of the spotlike pattern found in twin experiment II-(ii). Therefore, twin experiment III is set up to estimate only the initial state $\phi_{i}(0)$ with a fixed parameter $m=$ 0.1 . The true initial state $\phi_{i}^{\text {true }}(0)$ is assumed to be the phase field shown in Fig. 1(a). The other observational conditions
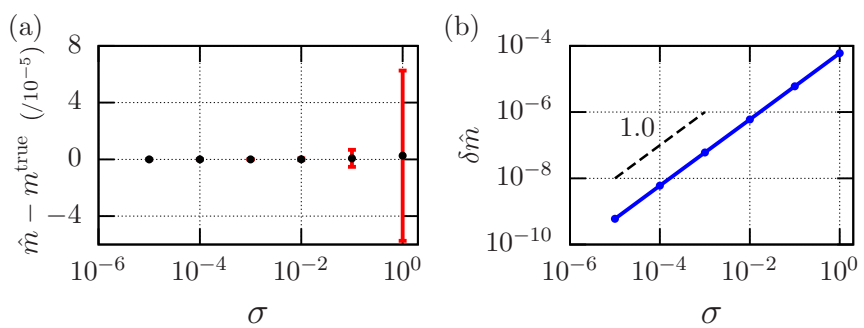

FIG. 4. Results of twin experiment I-(iii). The length of each error bar for optimum parameter $\hat{m}$ in (a) corresponds to the estimated uncertainty $\delta \hat{m}$ in (b). The dashed black line in (b) indicates a linear function. 

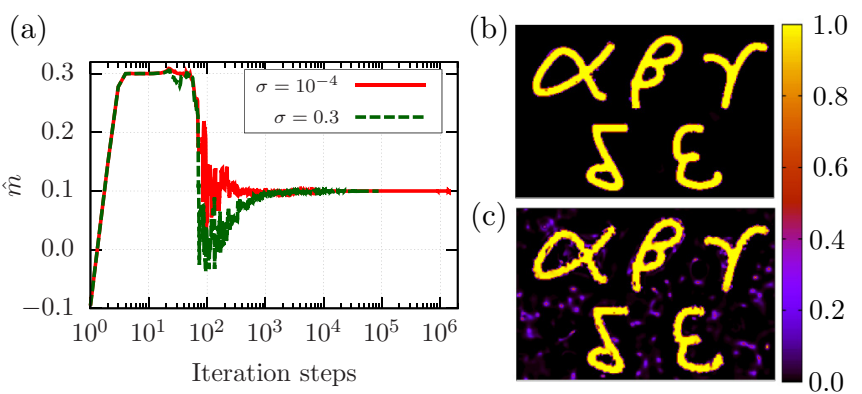

FIG. 5. Results of twin experiment II. (a) How the LBFGS method updates $m$ when the observation data noise has a small (solid red line) and large (dashed green line) standard deviation. (b) The optimum initial state of the phase field $\phi_{i}(0)$ in the case of small noise, and (c) that in the case of large noise.

are $T_{\min }=8.0 \tau, T_{\max }=30.0 \tau, \Delta T=0.1 \tau$, and $\sigma=0.3$, and the initial guess is $\phi_{i}(0)=0.2$.

Figures 6(a) and 6(b) show the estimated initial states after the 31 st and after the final iterations, respectively, and Fig. 6(c) shows how the cost function $J^{\prime}$ varies with the iteration. A spotlike pattern again appears in the estimated initial state [Fig. 6(b)] as the number of iterations increases. Note that the cost function $J^{\prime}$ is almost the same after the 31st step and after the final step, although Figs. 6(a) and 6(b) are much different.

This is caused by a feature inherent in the two-dimensional Kobayashi PF model. When a spot of radius $R_{0}$ evolves with time based on the PF model, whether it grows or decays depends on the relation between $m$ and $R_{0}$.

Figure 7 shows the phase diagram obtained by the twodimensional Kobayashi PF model under the assumption of axial symmetry. The destiny of a given spot depends on whether the radius is above or below the critical line, which means the critical radius is approximately inversely proportional to $m$ [43]. The radius of each spot in Fig. 6(b) is actually smaller than the critical radius, which is approximately $7.3 \epsilon$ in the case of $m=0.1$.

(a)

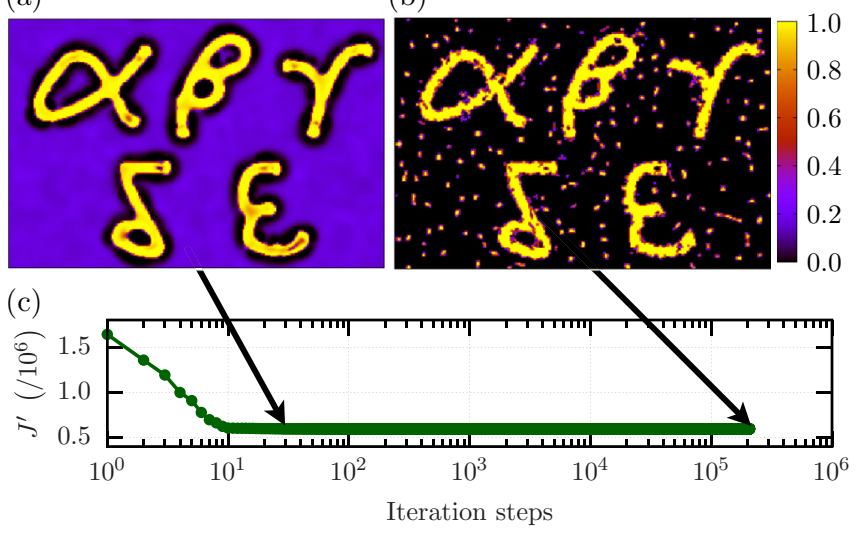

FIG. 6. Results of twin experiment III. Estimated initial states of the phase field $\phi_{i}(0)$ (a) after the 31st step and (b) after the final step in the iteration of the LBFGS method. (c) Improvement in the cost function $J^{\prime}$.

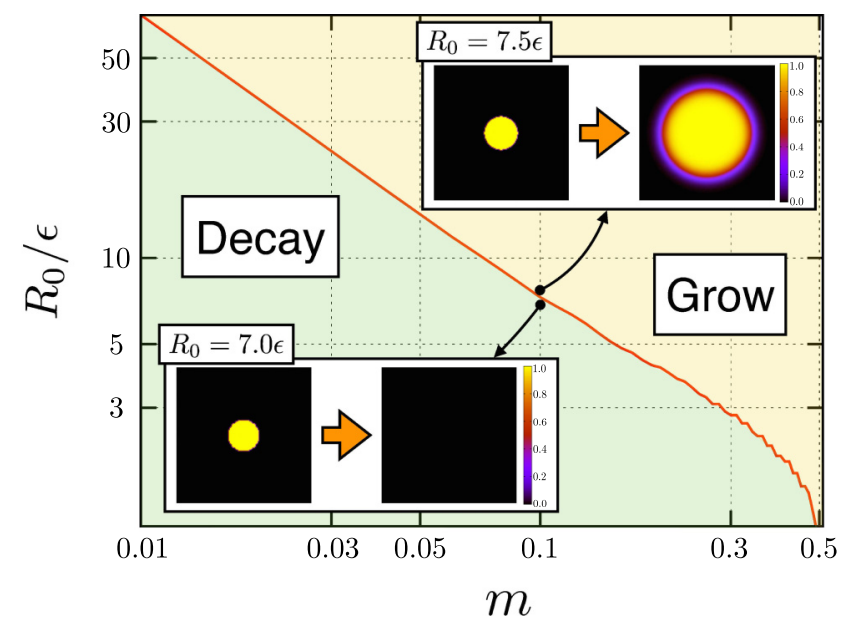

FIG. 7. Phase diagram for an axisymmetric two-dimensional Kobayashi PF model. The solid red line indicates the critical radius as a function of the parameter $m$. The region above or below the critical line corresponds to a spot growing or decaying with time, respectively.

Time evolutions starting from three different initial states are shown in Fig. 8. These results indicate that the phase fields at the time of the first observation, i.e., $t=8.0 \tau$, are completely coincident.

\section{CONCLUSIONS}

This paper described a new adjoint-based DA methodology applicable to massive autonomous models that not only determines the optimum estimates but also evaluates their uncertainties within a practical computation time and reasonable resource requirements. The uncertainties can be obtained as several diagonal elements of the inverse Hessian matrix, which is the covariance matrix of the normal distribution that approximates the posterior PDF in the neighborhood of the optimum estimates. The proposed approach adopts a second-order adjoint method, together with an appropriate iterative method, to compute the limited diagonal elements of

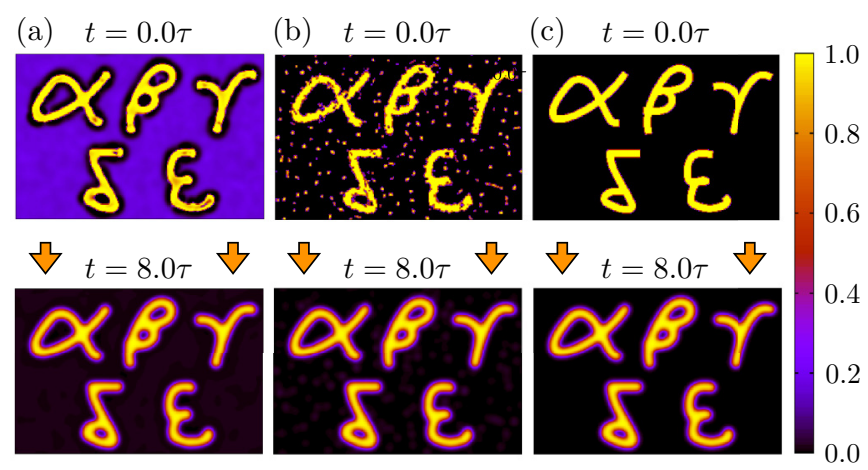

FIG. 8. Time evolutions of the phase fields starting from different initial states $\phi_{i}(0)$ : (a) the estimated initial state obtained after the $31 \mathrm{st}$ step [Fig. 6(a)], (b) that after the final step [Fig. 6(b)], and (c) the true initial state [Fig. 1(a)]. 
interest. Twin experiments using a two-dimensional Kobayashi PF model demonstrated the validity of the proposed method.

The uncertainties associated with physical quantities of interest depend on the quality and amount of data. Thus, conducting twin experiments prior to practical experiments allows us to determine how many observations are required to obtain the physical quantities of interest to the desired accuracy. Such feedback to practical experiments is already possible in systems with only a few degrees of freedom, but the proposed method makes this possible for massive simulation models.

This paper assumed that the true model was known, and the synthetic data in the twin experiments were generated based on the true model. This assumption is, of course, not appropriate in practical cases; a given "imperfect" simulation model usually generates a bias in its forecast unexplained by observation data. Such a bias due to the imperfectness of the model is called "model error." Evaluating the model error and investigating how it affects both the estimates and their uncertainties are important issues in adjoint-based DA, and some previous studies have proposed methodologies to evaluate model errors [44,45]. However, this issue is too complex to be discussed here, and re- mains controversial. As mentioned in Sec. I, this paper focuses on the methodology of quantifying uncertainties using the adjoint method within practical computational costs, which is important in many areas. The issue of the model error is beyond the scope of this paper, and will be considered in future work.

The proposed method is not only applicable to PF models, but to various models described by autonomous systems, e.g., shallow water equations, Navier's equations for elastic materials, and the Boltzmann equations. The proposed method is of great utility for evaluating the uncertainties of model parameters through DA, which is important in various fields of science, even when using massive models.

\section{ACKNOWLEDGMENTS}

This work was supported by Council for Science, Technology and Innovation (CSTI), Cross-ministerial Strategic Innovation Promotion Program (SIP), "Structural Materials for Innovation" (Funding agency: JST). The authors are grateful to Profs. Toshihiko Koseki, Tadashi Kasuya, Masato Okada, Munekazu Ohno, Kei Hirose, Peter Song, and Dr. Jonggyu Baek for useful discussions.
[1] R. Kobayashi, Physica D 63, 410 (1993).

[2] W. J. Boettinger, J. A. Warren, C. Beckermann, and A. Karma, Annu. Rev. Mater. Res. 32, 163 (2002).

[3] L.-Q. Chen, Annu. Rev. Mater. Res. 32, 113 (2002).

[4] Y. Tsukada, Y. Murata, T. Koyama, and M. Morinaga, Mater. Trans. 49, 484 (2008).

[5] T. Shimokawabe, T. Aoki, T. Takaki, T. Endo, A. Yamanaka, N. Maruyama, A. Nukada, and S. Matsuoka, in Proceedings of 2011 International Conference for High Performance Computing, Networking, Storage and Analysis, SC '11 (ACM, New York, NY, 2011), pp. 3:1-3:11.

[6] M. Ohno, T. Yamaguchi, D. Sato, and K. Matsuura, Comput. Mater. Sci. 69, 7 (2013).

[7] T. Takaki, ISIJ Int. 54, 437 (2014).

[8] D. Jacqmin, J. Comput. Phys. 155, 96 (1999).

[9] M. De Menech, Phys. Rev. E 73, 031505 (2006).

[10] H. G. Lee, K. Kim, and J. Kim, Int. J. Numer. Meth. Eng. 85, 1633 (2011).

[11] S. Reich and C. Cotter, Probabilistic Forecasting and Bayesian Data Assimilation (Cambridge University Press, Cambridge, 2015).

[12] E. Kalnay, Atmospheric Modeling, Data Assimilation and Predictability (Cambridge University Press, Cambridge, 2003).

[13] T. Tsuyuki and T. Miyoshi, J. Meteorol. Soc. Jpn. Ser. II 85B, 331 (2007).

[14] M. Ghil and P. Malanotte-Rizzoli, Advances in Geophysics (Elsevier, New York, 1991), Vol. 33, pp. 141-266.

[15] M. Kano, S. Miyazaki, Y. Ishikawa, Y. Hiyoshi, K. Ito, and K. Hirahara, Geophys. J. Int. 203, 646 (2015).

[16] T. Maeda, K. Obara, M. Shinohara, T. Kanazawa, and K. Uehira, Geophys. Res. Lett. 42, 7923 (2015).

[17] E. Motohashi, N. Isozaki, H. Nagao, and T. Higuchi, Operations Research 57, 574 (2012) (in Japanese).

[18] K. Sasaki, A. Yamanaka, S. Ito, and H. Nagao (unpublished).

[19] G. Evensen, Ocean Dynam. 53, 343 (2003).
[20] P. L. Houtekamer and H. L. Mitchell, Mon. Weather Rev. 126, 796 (1998).

[21] G. Ueno, T. Higuchi, T. Kagimoto, and N. Hirose, SOLA 3, 5 (2007).

[22] G. Kitagawa, Introduction to Time Series Modeling, Chapman \& Hall/CRC Monographs on Statistics \& Applied Probability (CRC Press, Boca Raton, FL, 2010).

[23] A. Doucet, S. Godsill, and C. Andrieu, Stat. Comput. 10, 197 (2000).

[24] H. Nagao, T. Higuchi, S. Miura, and D. Inazu, Comput. J. 56, 355 (2013).

[25] J. M. Lewis and J. C. Derber, Tellus A 37A, 309 (1985).

[26] F.-X. Le Dimet and O. Talagrand, Tellus A 38A, 97 (1986).

[27] M. Iri and K. Kubota, The Japan Society for Industrial and Applied Mathematics 1, 17 (1991) (in Japanese).

[28] D. N. Daescu, Mon. Weather Rev. 136, 3050 (2008).

[29] V. P. Shutyaev, F.-X. Le Dimet, V. I. Agoshkov, and E. I. Parmuzin, Izv. Atmos. Ocean. Phys. 51, 342 (2015).

[30] W. C. Thacker, J. Geophys. Res-Oceans 94, 6177 (1989).

[31] F. Rabier and P. Courtier, Q. J. Roy. Meteor. Soc. 118, 649 (1992).

[32] I. Y. Gejadze, F.-X. L. Dimet, and V. Shutyaev, SIAM J. Sci. Comput. 30, 1847 (2008).

[33] I. Y. Gejadze, G. Copeland, F.-X. Le Dimet, and V. Shutyaev, J. Comput. Phys. 230, 7923 (2011).

[34] I. Y. Gejadze, V. Shutyaev, and F.-X. L. Dimet, Q. J. Roy. Meteor. Soc. 139, 1826 (2013).

[35] J. Nocedal, Math. Comput. 35, 773 (1980).

[36] Z. Wang, I. Navon, F. Le Dimet, and X. Zou, Meteorol. Atmos. Phys. 50, 3 (1992).

[37] Z. Wang, K. Droegemeier, and L. White, Comput. Optim. Appl. 10, 283 (1998)

[38] F.-X. Le Dimet, I. Navon, and D. N. Daescu, Mon. Weather Rev. 130, 629 (2002). 
[39] I. S. Aranson, V. A. Kalatsky, and V. M. Vinokur, Phys. Rev. Lett. 85, 118 (2000)

[40] R. Spatschek, M. Hartmann, E. Brener, H. Müller-Krumbhaar, and K. Kassner, Phys. Rev. Lett. 96, 015502 (2006).

[41] S. Komura and Y. Yamazaki, J. Phys. Soc. Jpn. 76, 083801 (2007).
[42] L. Armijo, Pacific J. Math. 16, 1 (1966).

[43] M. Castro, Phys. Rev. B 67, 035412 (2003).

[44] P. Vidard, A. Piacentini, and F.-X. Le Dimet, Tellus A 56, 177 (2004).

[45] V. Shutyaev, A. Vidard, F.-X. Le Dimet, and I. Gejadze, Russ. J. Numer. Anal. M. 31, 105 (2016). 